\title{
Impact of Mass Transpiration on Unsteady Boundary Layer Flow of Impulsive Porous Stretching
}

\author{
Koratageri Revanna Nagaraju ${ }^{1}$, Ulavathi Shettar Mahabaleshwar ${ }^{2 *}$, Asimina A. Krimpeni ${ }^{3}$, Ioannis E. Sarris ${ }^{3}$, Giulio Lorenzini ${ }^{4}$ \\ ${ }^{1}$ Department of Mathematics, Government Engineering College, Hassan 573 201, India \\ ${ }^{2}$ Dept. of Mathematics, Davangere University, Shivagangotri, Davangere 577 007, India \\ ${ }^{3}$ Department of Mechanical Engineering, University of West Attica, 12244 Athens, Greece \\ ${ }^{4}$ Department of Engineering and Architecture, University of Parma, Parco Area Delle Scienze 181/A, 43124 Parma, Italy
}

Corresponding Author Email: ulavathi@gmail.com

https://doi.org/10.18280/mmep.060305

Received: 17 July 2019

Accepted: 11 August 2019

\section{Keywords:}

darcy number, ADM, Mass

suction/injection, pade approximants

\begin{abstract}
Detailed The analytical solution of the unsteady boundary layer flow due to impulsive porous stretching sheet is solved by means of an Adomian Decomposition Method (ADM). The ADM alongside Pade approximants are connected to tackle the nonlinear partial differential equation with different boundary conditions got in demonstrating the unsteady boundary layer flow. Apparently, this yields better accuracy when compared to results from other prime methods. The present results are in good match with the pioneering work of other authors. The impact of parameters like mass suction/injection parameter and Darcy number on the velocity profile were examined. The present results can be found very useful in a wide range of applications in extrusion process and related process in fluid dynamics and heat transfer problems.
\end{abstract}

\section{INTRODUCTION}

Throughout In-depth study of unsteady, laminar boundary layer flow due to a stretching media, as well as its industrial applications, is of prominent interest in the classical fluid dynamics field. Production of sheet materials has risen in a numerous of industrial manufacturing applications and hitherto involves both metal and polymer sheets. The material is in a molten phase when extruded through a die, then it cools and solidifies.

Other similar examples are:

(1) Cooling of a large metallic plate in a bath,

(2) Glass blowing,

(3) Continuous casting,

(4) Fiber spinning.

As implementations in industry become wider and more sophisticated, the study of theoretical fluid mechanics phenomena involving not only steady but also unsteady laminar boundary layer is needed.

In the last few decades, flow studies in porous media has drawn researchers attention due to its increasing applications in engineering and in advanced state-of-the-art technology. Porous media is defined as a mixture of fluid and solid -the base material is usually a solid- that have interconnected pores through or over which the fluid can move. In porous media problems, the most common analytical approach is delivered by using Darcy theory [1]. Important effects due to the porosity may appear in porous media as analysed by Kaviany [2]. Intensive work has be presented in a variety of articles about porous media and thermal analysis of flow by Starov and Zhdanov [3] and Tamayol et al. [4], both in a stretching sheet and wall of either a Newtonian or non-Newtonian fluid (Mahabaleshwar [5], Hayat et al. [6], Prasad et al. [7]), as well as by considering the effect of magnetic fields (El-Mistikawy et al [8], Pand et al. [9]). The outcome of the present study has various applications, as it may be implemented in fields, like bio-fluid dynamics, hydrodynamics, aeronautics physics and others. Flow and heat transfer laws in the boundary layer induced by a stretching surface are implemented in manufacturing processes, such as in the cooling of metallic plates, glasses and polymers, aerodynamic extrusion of plastic sheets, boundary layer along a liquid film and condensation process. Crane [10] was a pioneer and investigated the flow caused by sheet stretching. Gupta and Gupta [11] and many other researchers relied upon Crane's work and extended it by including the effect of heat and mass transfer analysis under different physical states.

A significant part in literature deals with the study of boundary layer flow over unsteady stretching surfaces as in Wang [12], and Chen [13], while Magyari and Keller [14] focus on heat and mass transfer on boundary layer flow owing to an exponentially continuous stretching sheet. On the other hand, viscous flow due to a shrinking sheet was studied by Miklavcic and Wang [15], as well as stagnation flow towards a shrinking sheet was introduced by Wang [16]. Other researchers investigate linear and exponential stretching of a material (usually plastic) [14], whereas in Kumaran and Ramanaiah [17] the boundary layer fluid flow of a general quadratic stretching sheet has been assumed. Many articles reffer to the impulsive motion of a surface in a viscous fluid (Stewartson [18-19], Wang et al. [20]) and it has been proven that when $t>1$ flow goes through an transition from Rayleigh flow to Blasius flow. It is significant, though, to stress out that the solution approach is either analytical or numerical in all the above works.

In order to obtain an appropriate solution Adomian ([21 22]) founded the Adomian Decomposition Method (ADM), which enables the accurate and efficient analytic solution of nonlinear ordinary or partial differential equations avoiding linearization or perturbation approaches. This method unifies 
the treatment of linear and nonlinear, ordinary or partial differential equations, or systems of such equations, into a single basic method, which is applicable to both initial and boundary-value problems. Due to its effectiveness, the method has been studied and has been used by many researchers (Biazar and Shafiot [23], Cherruault [24], Cherruault and Adomian [25], Disu and Ajibola [26]) in a variety of problems.

The objective of the present work is to present an analytical solution of the unsteady boundary layer flow of a viscous and incompressible fluid due to an impulsively stretching sheet over a porous media. The unsteadiness in the flow is caused by impulsively creating motion in the free stream and simultaneously porous stretching the surface. The boundary layer approximation Navier-Stokes equation will be solved by using the Adomian decomposition method along with Pade approximants (Baker [27]), Boyd [28]). Pade approximants is the "best" approximation of a function by a rational function of given order. It is worth mentioning that under this technique, the approximant's power series agrees with the power series of the function which is approximating.

\section{MATHEMATICAL FORMULATION GOVERNING EQUATIONS}

In the present article, the unsteady, Newtonian, laminar incompressible fluid flow of an impulsive porous stretching sheet is considered. Le $\psi$ is the stream function, $u$ and $v$ are velocity components in $x$ and $y$ directions, respectively that are given by $u=\frac{\partial \psi}{\partial y}$ and $v=-\frac{\partial \psi}{\partial x}, \quad \mathcal{C}$ is stretching rate $\left(s^{-1}\right)$ i.e. a positive constant in the present case, $k$ is the permeability parameter, $\eta$ and $\xi$ are the transformed dimensionless variables, $v$ is the kinematic velocity, $t$ is the time and $\tau$ is the normalized time through coefficient $\alpha$ (the non-dimensional time), and $v_{w}$ is the stretching velocity of the sheet.

The imposed governing nonlinear equations are as follows:

$$
\begin{gathered}
\frac{\partial u}{\partial x}+\frac{\partial v}{\partial y}=0 \\
\frac{\partial u}{\partial t}+u \frac{\partial u}{\partial x}+v \frac{\partial u}{\partial y}=\frac{\partial^{2} u}{\partial y^{2}}-\frac{v}{\kappa} u
\end{gathered}
$$

The corresponding boundary conditions are

$$
\begin{aligned}
& u=c x \text { and } v=v_{w} \text { when } t=0 \text { and } y=0, \\
& u=v=0 \text { when } t=0 \text { and } y>0, \\
& u=c x \text { and } v=0 \text { when } t>0 \text { and } y=0, \\
& u=0 \text { as } y \rightarrow \infty .
\end{aligned}
$$

The considered similarity transformations are as found in Williams and Rhyne [29]

$$
\psi=\sqrt{c \nu \xi} f(\eta, \xi) \text { and } \eta=\sqrt{\frac{c}{\nu \xi}} y, \xi=1-e^{-\tau}, \tau=a t
$$

The reduced non-dimensional Navier-Stokes equation is then

$$
\begin{aligned}
& \mathrm{f}_{\eta \eta \eta}+\frac{1}{2} \eta(1-\xi) f_{\eta \eta}+\xi\left(f f_{\eta \eta}-f_{\eta}^{2}\right) \\
& -\xi(1-\xi) f_{\eta \xi}-\xi D a^{-1} f_{\eta}=0 .
\end{aligned}
$$

where, $D a^{-1}=\frac{v}{c k}$ is the porous media parameter (i.e. the inverse Darcy number).

The reduced boundary conditions are:

$$
f(0, \xi)=V_{c}, f_{\eta}(0, \xi)=1 \text { and } f(\infty, \xi)=0 .(6 \mathrm{a}, \mathrm{b}, \mathrm{c})
$$

where, $V_{c}=-\frac{v_{w}}{\sqrt{c / v \xi}}$ is the suction/injection parameter.

An analytical solution of Eq. (5) is time dependent as it is third order nonlinear partial differential equation with inhomogeneous boundary conditions. The solution is obtained using the ADM with Pade approximants. The ADM offers an approximate analytical solution expressed in terms of converging power series. Fewer iterations performed in ADM are needed, so as to compute an approximate analytical solution in the form of power series and then Pade approximants are used to handle the limit at $\eta \rightarrow \infty$, at which the value of unknown $\alpha=f_{\eta \eta}(0)$ is computed. For the case $\xi=0$, the Eq. (5) converted into classical Rayleigh type equation:

$$
f_{\eta \eta \eta}+\frac{1}{2} \eta f_{\eta \eta}=0
$$

The exact solution is

$$
f(\eta)=-\frac{e^{-\frac{\eta^{2}}{4}}\left(2-2 e^{\frac{\pi^{2}}{4}}-e^{\frac{\pi^{2}}{4}} \sqrt{\pi \eta}+e^{\frac{\pi^{2}}{4}} \sqrt{\pi \eta} \operatorname{Erf}\left[\frac{\eta}{2}\right]\right)}{\sqrt{\pi}}
$$

Under the assumption that $\xi=1, \tau \rightarrow \infty$, Eq. (5) reduces to classical Crane type equation:

$$
f_{\eta \eta \eta}+f f_{\eta \eta}-f_{\eta}^{2}-D a^{-1} f_{\eta}=0
$$

The exact solution of Eq. (9), implementing the boundary conditions of Eq. (6), be formed as:

$$
f(\eta)=V_{c}+\frac{1-e^{-\beta \eta}}{\beta} .
$$

In the absence of porosity (i.e. inverse Darcy number $D a^{-1}$ is zero) and no suction/injection (i.e. the parameter $V_{c}$ is also zero), the exact solution of Eq. (9) can be written (Crane [10]):

$$
f(\eta)=1-e^{-\eta}
$$

\section{ADOMIAN DECOMPOSITION METHOD (ADM)}

The Adomian decomposition method is applied to solve Eq. (5) with Eq. (6) and provides the relaxation: 


$$
L f=\xi\left(f_{\eta}^{2}-f f_{\eta \eta}\right)-\frac{1}{2} \eta(1-\xi) f_{\eta \eta}+\xi(1-\xi) f_{\eta \xi}+\xi D a^{-1} f_{\eta}
$$

where $L=\frac{\partial^{3}}{\partial \eta^{3}}$ is the third order differential operator.

By applying the inverse operator $L^{-1}(*)=$ $\int_{0}^{\theta} \int_{0}^{\theta} \int_{0}^{\theta}(*) d \theta d \theta d \theta$ to $\operatorname{Eq}(12)$ with the different boundary conditions of (Eq. (6)), the solution $f_{0}=V_{c}+\eta+\frac{1}{2} \alpha \eta^{2}$ is obtained, as well as the approximate solution $f(\eta, \xi)=$ $\sum_{m=0}^{\infty} f_{m}(\eta, \xi)$. Thus, in the unknown quantity $\alpha=f_{\eta \eta}(0, \xi)$ is found. Then, the exact analytical solution owing to ADM is obtained as:

$$
\begin{aligned}
f & =V_{c}+\eta+\frac{1}{2} \alpha \eta^{2}+L^{-1}\left\{\xi\left(f_{\eta}^{2}-f f_{\eta \eta}\right)\right. \\
& \left.-\frac{1}{2} \eta(1-\xi) f_{\eta \eta}+\xi(1-\xi) f_{\eta \xi}+\xi D a^{-1} f_{\eta}\right\}
\end{aligned}
$$

The ADM iteration process then:

$$
\begin{gathered}
f_{0}=V_{c}+\eta+\frac{1}{2} \alpha \eta^{2} \\
f_{m+1}=L^{-1}\left\{\begin{array}{c}
\xi(1-\xi) f_{\eta \xi}-\frac{1}{2} \eta(1-\xi) f_{\eta \eta}+\xi A_{m}+ \\
\left.D a^{-1} f_{\eta}\right\} \text { for } m \geq 0
\end{array}\right.
\end{gathered}
$$

where

$$
\begin{gathered}
A_{0}=f_{0}^{\prime 2}-f_{0} f_{0}^{\prime \prime} \\
A_{m}=\left\{\begin{array}{cc}
2 \sum_{i=0}^{\left(\frac{m-1}{2}\right)} f_{i}^{\prime} f_{m-i}^{\prime}-\sum_{i=0}^{m} f_{i} f_{m-i}^{\prime \prime} & \text { if } m \text { is odd } \\
\left(\frac{m-2}{2}\right) & \\
2 \sum_{i=0}^{m} f_{i}^{\prime} f_{m-i}^{\prime}+f_{m / 2}^{\prime 2}-\sum_{i=0}^{m} f_{i} f_{m-i}^{\prime \prime} & \text { if } m \text { is even }
\end{array}\right.
\end{gathered}
$$

With the help of Mathematica (Wellin [30] built-in package software, the approximate solution $\varphi_{n}=\sum_{n=0}^{\infty} f_{\eta}$ is obtained. The first three terms of the solution are listed below.

$$
\begin{gathered}
f_{0}=V_{c}+\eta+\frac{1}{2} \alpha \eta^{2} \\
f_{1}=-\frac{1}{48} \alpha \eta^{4}+\frac{1}{6} \xi \eta^{3}+\frac{1}{6} D a^{-1} \xi \eta^{3}-\frac{1}{6} V_{c} \alpha \xi \eta^{3} \\
+\frac{1}{16} \alpha \xi \eta^{4}+\frac{1}{24} D a^{-1} \alpha \xi \eta^{4}+\frac{1}{120} \alpha^{2} \xi \eta^{5} \\
f_{2}=\frac{1}{960} \alpha \eta^{6}+\frac{1}{240} V_{c} \alpha \xi \eta^{5}-\frac{1}{720} \alpha \xi \eta^{6}-\frac{1}{720} D a^{-1} \alpha \xi \eta^{6} \\
-\frac{1}{3360} \alpha^{2} \xi \eta^{7}-\frac{1}{24} V_{c} \xi^{2} \eta^{4}-\frac{1}{24} D a^{-1} V_{c} \xi^{2} \eta^{4} \\
+\frac{1}{24} V_{c}^{2} \alpha \xi^{2} \eta^{4} \\
+\frac{1}{120} D a^{-1} \xi^{2} \eta^{5}+\frac{1}{120}\left(D a^{-1}\right)^{2} \xi^{2} \eta^{5}-\frac{1}{80} D a^{-1} \alpha \xi^{2} \eta^{5} \\
-\frac{1}{60} D a^{-1} V_{c} \alpha \xi^{2} \eta^{5}+\frac{1}{576} \alpha \xi^{2} \eta^{6}+\frac{1}{240} D a^{-1} \alpha \xi^{2} \eta^{6} \\
+\frac{1}{720}\left(D a^{-1}\right)^{2} \alpha \xi^{2} \eta^{6}-\frac{1}{240} V_{c} \alpha^{2} \xi^{2} \eta^{6}+\frac{1}{10080} \alpha^{2} \xi^{2} \eta^{7}
\end{gathered}
$$

$$
+\frac{1}{2520} D a^{-1} \alpha^{2} \xi^{2} \eta^{7}-\frac{1}{40320} \alpha^{2} \xi^{2} \eta^{8}
$$

and so on

\section{PADE APPROXIMANTS}

Frequently, the power series can be considerably accelerated in moderation by being rearranged into a ratio of such two expansions:

$$
P_{M}^{L}(x)=\frac{\sum_{i=0}^{L} a_{i} x^{i}}{\sum_{i=0}^{M} b_{i} x^{i}}
$$

It is considered, without loss of generality, that $b_{0}=1$ and generalization of the series expansion with degrees $(L=M)$

$$
T_{L, M}(x)=\sum_{i=0}^{L+M} c_{i} x^{i}
$$

From Eq. (18) and Eq. (19), we have

$$
c_{0}+c_{1} x+c_{2} x^{2}+\ldots . .=\frac{a_{0}+a_{1} x+a_{2} x^{2}+\ldots}{b_{0}+b_{1} x+b_{2} x^{2}+\ldots .}
$$

From Eq. (20), by cross multiplication, we find that

$$
\begin{gathered}
a_{0}=c_{0} \\
a_{1}=c_{1}+c_{0} b_{1} \\
a_{2}=c_{2}+c_{1} b_{1}+c_{0} b_{2}
\end{gathered}
$$

$[L / M]$ approximant construction depends on choosing the degree of both numerator $(L)$ and denominator $(M)$. Pade approximant is an effective tool in handling boundary conditions in infinite domains. Thus, the diagonal approximants are constructed, being the most accurate approximant when $[L=M]$, by using the boundary condition $\operatorname{Lim}_{\eta \rightarrow \infty} f=0$ to evaluate $\alpha=f_{\eta \eta}(0)$.

Table 1. Association of Pade approximants for various values of $\mathrm{L}$ and $\mathrm{M}$ numbers, $a=f_{\eta \eta}(0)$ and when $\xi=1$ and

$$
V_{c}=0
$$

\begin{tabular}{ccccc}
\hline $\boldsymbol{\eta}$ & {$[\mathbf{1 0} / \mathbf{1 0}]$} & {$[\mathbf{1 5 / 1 5}]$} & {$[\mathbf{2 0 / 2 0}]$} & {$[\mathbf{2 5 / 2 5}]$} \\
\hline 6 & -0.564259 & -0.564203 & -0.564202 & -0.564202 \\
7 & -0.564604 & -0.564205 & -0.564190 & -0.564190 \\
8 & -0.565821 & -0.564297 & -0.564191 & -0.564190 \\
9 & -0.568599 & -0.564666 & -0.564200 & -0.564190 \\
10 & -0.573489 & -0.565678 & -0.564245 & -0.564189 \\
11 & -0.580757 & -0.567810 & -0.564394 & -0.564185 \\
\hline
\end{tabular}

Results of the present Pade approximants dependence on the calculation of $a=f_{\eta \eta}(0)$ for various $\eta$ and $D a^{-1}$ values can be found in Tables 1, 2 and 3, also in comparison with 
analytical solutions or the results of Refs. [31, 32, 33]. As it is observed in Table 1, the present method converged fairly well as the values of $[L / M]$ in Pade approximants diversify, for $\xi=1$ and $V_{c}=0$ as as $D a^{-1}$ is increased.

Table 2. Comparison of the present results against the exact one for different values of $D a^{-1}$ for the calculation of $a=$ $f_{\eta \eta}(0)$ and when $\xi=1$ and $V_{c}=0$

\begin{tabular}{ccc}
\hline $\boldsymbol{D a}^{-\mathbf{1}}$ & $\begin{array}{c}\text { Exact } \\
\text { results }\end{array}$ & $\begin{array}{c}\text { Present } \\
\text { results }\end{array}$ \\
\hline 0 & -1.000000 & -1.000194 \\
0.5 & -1.224740 & -1.225161 \\
1 & -1.414213 & -1.414184 \\
\hline
\end{tabular}

Moreover, the present method is compared, for various $D a^{-1}$ numbers, against the exact solution of Eq. (11) as Table 2 is showing, $\mathrm{Da}^{-1}$ dependence on the results can be captured, also, very well.

Table 3. Comparison of $a=f_{\eta \eta}(0)$ calculation for different $\xi$ values in the absence of $D a^{-1}$ and $V_{c}$ against results from Refs. [31-33]

\begin{tabular}{ccccc}
\hline$\xi$ & Ref. [31] & Ref. [32] & Ref. [33] & $\begin{array}{c}\text { Present } \\
\text { resuts }\end{array}$ \\
\hline 0 & -0.564189 & -0.564189 & -0.564374 & -0.564189 \\
0.5 & -0.790412 & -0.801820 & -0.800412 & -0.790741 \\
0.8 & -0.920056 & -0.925270 & -0.920055 & -0.920470 \\
0.9 & -0.962333 & -0.963376 & -0.962340 & -0.961664 \\
1 & -1.000000 & -1.000000 & -1.000000 & -1.000194 \\
\hline
\end{tabular}

Finally, results from the present method are compared for various $\xi$ against Refs. [31-33] for the calculation of $\xi$ in the case of $D a^{-1}=V_{c}=0$ are very accurate. Since the present method is found adequate to calculate Eq. (13), the unsteady flow, the impulsive porous stretching sheet will be analyzed in the next section.

\section{RESULTS AND DISCUSSION}

Using the approximate series, solution of the function $f(\eta, \xi)$ and its derivative $f(\eta, \xi)$ are determined Adomian Decomposition Method. Incorporating the condition at infinity, the unknown $a=f_{\eta \eta}(0)$ is determined by applying the Pade approximants $[L / M]$ while following the procedure proposed by Boyd [28]. The numerical results of $a=f_{\eta \eta}(0)$ are listed in Table 1 for various values of $\eta$ An excellent match is found with the exact value $-\frac{1}{\sqrt{\pi}}=-0.564190$ for $\xi=0$ in the absence of $D a^{-1}$ and $\mathrm{V}_{\mathrm{c}}$ parameters as $\mathrm{L}$ and $\mathrm{M}$ increase. Comparison of the results against the exact solution of $f(\eta)$ and $f_{\eta}(\eta)$ are depicted in Figure 1 and Figure 2, respectively, for the cases $\xi=0$ and $\xi=1$ in the absence of the parameters $D a^{-1}$ and $V_{c}$ proving that they are in excellent agreement. Besides the comparison between the present results and the exact solution, the temporal evolution of the transverse and the axial velocity profiles are presented as $\eta$ increases from the initial time of $\xi=0$ to the equilibrium time at $\xi=1$ where steady-state conditions are found. This temporal evolution of the transverse velocity component gives also inputs about the development of the boundary layer of the film and the evolution of its thickness as it will be discussed below.

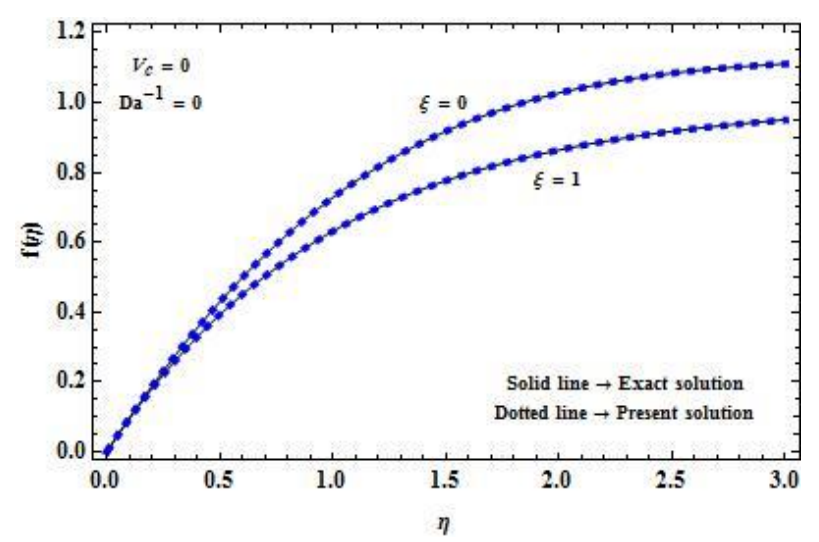

Figure 1. Transverse velocity profile $f(\eta)$ in respect to $\eta$, for $\xi=0$ and $\xi=1$ with $V_{c}=0$ and $D a^{-1}=0$

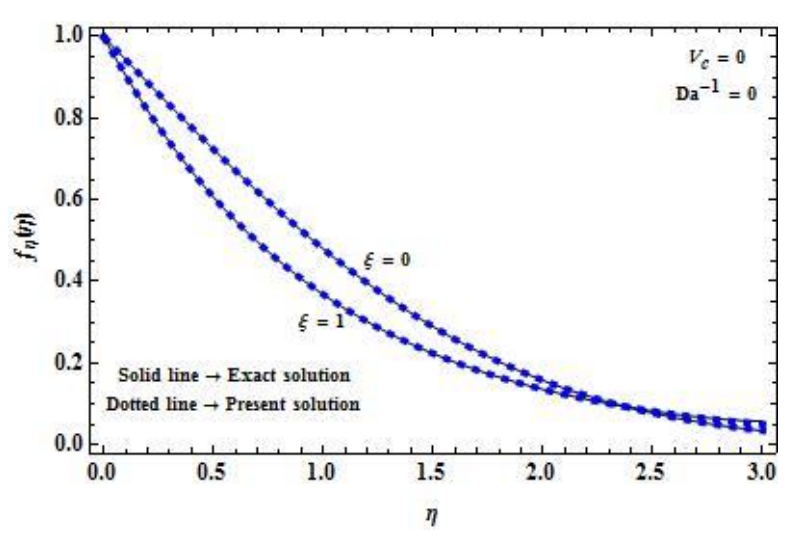

Figure 2. Axial velocity profile $f_{\eta}(\eta)$ in respect to $\eta$ for $\xi=$ 0 and $\xi=1$ with $V_{c}=0$ and $D a^{-1}=0$

The effect of $D a^{-1}$ in the two velocity components $f(\eta)$ and $f_{\eta}(\eta)$ are shown in Figures 3 and 4, respectively, for $\xi=$ 1 and $D a^{-1}=0$ As porosity increases, the fluid flows more difficult and thus both velocity components are reduced. The most significant effect of porosity increase is found on the transverse velocity since axial momentum is driven by the fixed wall motion and its decrease due to porosity is transferred immediately transversally. Porosity increase has also a significant influence on the thickness of the boundary layer as it is shown in Figure 4. Thus, as $D a^{-1}$ increases and the flow is slowed due to porosity increase, the boundary layer thickness is reduced.

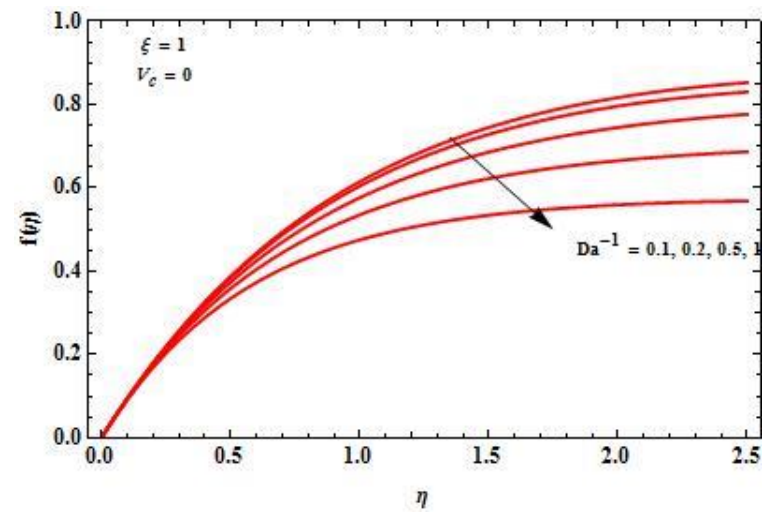

Figure 3. Transverse velocity profile $f(\eta)$ in respect to $\eta$ for different values of $D a^{-1}$ with $V_{c}=0$ and $\xi=1$ 


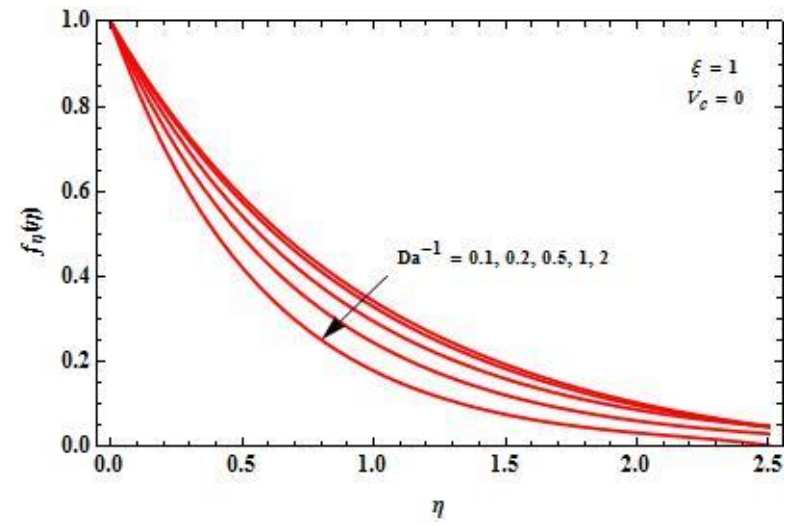

Figure 4. Axial velocity profile $f_{\eta}(\eta)$ in respect to $\eta$ for different values of $D a^{-1}$ with $V_{c}=0$ and $\xi=1$

As the stretching velocity of the sheet increases from $V_{c}=$ -0.5 to 0.2 for the equilibrium conditions at $\xi=1$ and for $D a^{-1}=0.5$, the transverse velocity profiles are following the same distribution along $\eta$, with its magnitude to be linearly decreased as $V_{c}$ decreases. The boundary value of the transverse velocity is determined by the boundary condition $V_{c}$ at $\eta=0$, while the velocity is developed with increased slope as $V_{c}$ increases.

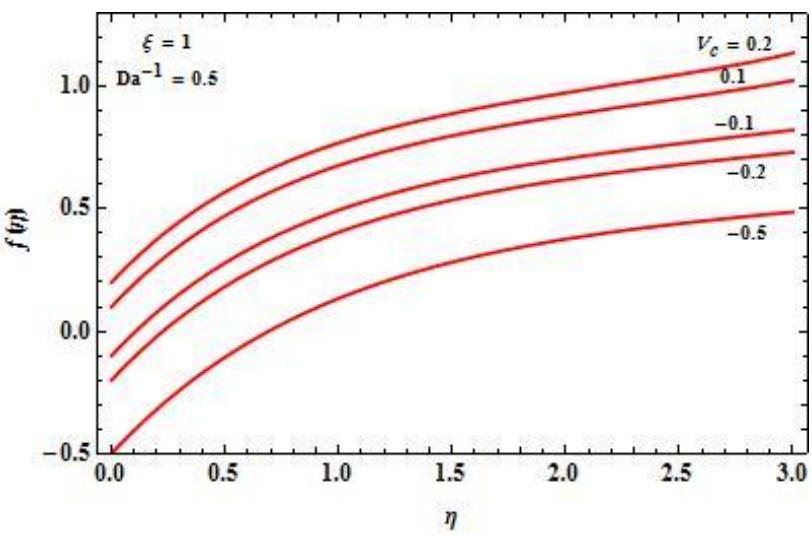

Figure 5. Transverse velocity profile $f(\eta)$ in respect to $\eta$ for different values of $V_{c}$ with $D a^{-1}=0.5$ and $\xi=1$

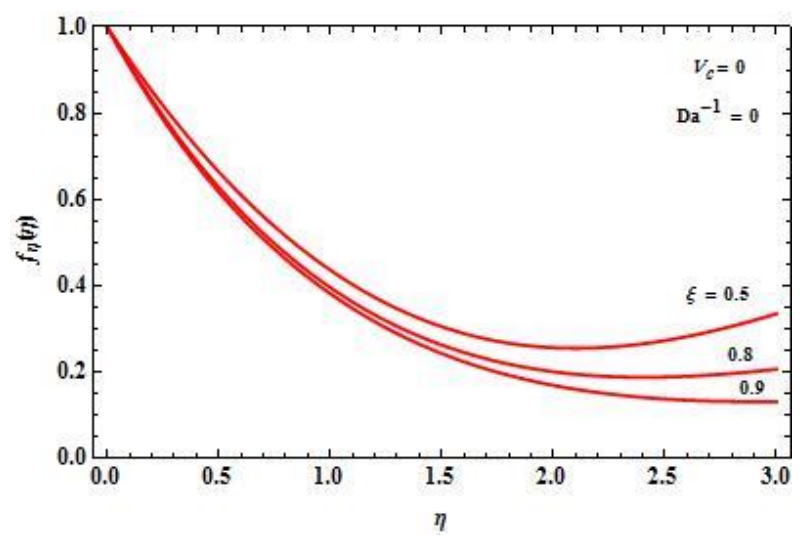

Figure 6. Axial velocity profile $f(\eta)$ in respect to $\eta$ for different values of $\xi$ with $D a^{-1}=0$ and $V_{c}=0$

The temporal effect on the velocity profile is shown in Figure 6 for the axial velocity component $f_{\eta}(\eta)$ for various $\xi$ and for $D a^{-1}=0$ and $V_{c}=0$ The transverse velocity component is less sensitive to temporal variations since it is the flow driven direction. As the surface of the film is under stronger transient conditions the most important temporal effects are found for $\eta>1.5$ while for $\eta<1.5$ no significant temporal effects are encountered due to the stabilized action of the fixed wall suction/injection parameter. Thus, the thickness of the boundary layer is progressively increased as $V_{c}$ increases until it is found its equilibrium value for $\xi=1$.

\section{CONCLUSIION}

With the proposed methodology, a sound analytical series solution using ADM with Pade approximants is obtained for the film porous flow in a stretching sheet that is compared successfully to the solutions obtained by other authors for the cases $0<\xi<1$ as well as $\xi=0$ and $\xi=1$. As $\xi$ increases, the ratio of the axial $f_{\eta}(\eta, \xi)$ to transverse $f(\eta, \xi)$ velocity is found to increase and, thus, laminar boundary layer thickness decreases. Moreover, a large variation in the velocity profile away from the surface of the sheet can be extracted from the method application as the inverse Darcy number and the suction/injection parameter varied.

\section{ACKNOWLEDGMENT}

Author Nagaraju K R would like to thank his beloved Vice Chancellor Dr. Karisiddappa, VTU, Belagavi (Former Principal of Government Engineering College, Hassan) for his valuable support.

\section{REFERENCES}

[1] Kim, S.J., Jang, S.P. (2002). Effects of the Darcy number, the Prandtl number, and the Reynolds number on local thermal non-equilibrium. International Journal of Heat and Mass Transfer, 45(19): 3885-3896. https://doi.org/10.1016/S0017-9310(02)00109-6

[2] Kaviany, M. (1992). Principles of heat transfer in porous media, Springer, New York. https://doi.org/10.1007/9781-4612-4254-3

[3] Starov, V.M., Zhdanov, V.G. (2001). Effective viscosity and permeability of porous media. Colloids Surfaces A: Physicochemical and Engineering Aspects, 192(1-3): 363-375.

[4] Tamayol, A., Hooman, K., Bahrami, M. (2010). Thermal analysis of flow in a porous medium over a permeable stretching wall. Transport in Porous Media, 85(3): 661676. https://doi.org/10.1007/s11242-010-9584-X

[5] Mahabaleshwar, U.S. (2013). Linear stretching sheet problem with suction in porous medium. Open Journal of Heat, Mass and Momentum Transfer, 1(1): 13-18.

[6] Hayat, T., Javed, T., Abbas, Z. (2008). Slip flow and heat transfer of a second grade fluid past a stretching sheet through a porous space. International Journal of Heat and Mass Transfer, 51: 4528-4534. https://doi.org/10.1016/j.ijheatmasstransfer.2007.12.022

[7] Prasad, K.V., Abel, S., Datti, P.S. (2003). Diffusion of chemically reactive species of a non-Newtonian fluid immersed in a porous medium over a stretching sheet. International Journal of Non-Linear Mechanics, 38(5): 651-657. https://doi.org/10.1016/S0020-7462(01)001226 
[8] El-Mistikawy, T.M.A., El-Fayez, F.M.N. (2013). Limiting Behavior of MHD flow over a porous rotating disk with Hall currents, ZAMM. Journal of Applied Mathematics and Mechanics, 93(9): 706-712. https://doi.org/10.1002/zamm.201200065

[9] Panda, J.P., Dash, N., Dash, G.C. (2012). Heat and mass transfer on MFD flow through porous media over an accelerating surface in the presence of suction and blowing. Journal of Engineering Thermophysics, 21(2): 119-130. https://doi.org/10.1134/s1810232812020038

[10] Crane, L.J. (1970). Flow past a stretching plate. Zeitscrift. Für Angewandte Mathematik Und Physik ZAMP, 21(4): 645-647.

[11] Gupta, P.S., Gupta, A.S. (1977). Heat and mass transfer on a stretching sheet with suction or blowing. The Canadian Journal of Chemical Engineering, 55(6): 744746. https://doi.org/10.1002/cjce.5450550619

[12] Wang, C.Y. (1990). Liquid film on an unsteady stretching sheet. Quarterly of Applied Mathematics, 48 (4): 601-610.

[13] Chen, C.H. (2006). Effect of viscous dissipation on heat transfer in a non-Newtonian liquid film over an unsteady stretching sheet. Journal of Non-Newtonian Fluid Mechanics, 135(2-3): 128-135 https://doi.org/10.1016/j.jnnfm.2006.01.009

[14] Magyari, E., Keller, B. (1999). Heat and mass transfer in the boundary layers on an exponentially stretching continuous surface. Journal of Physics D: Applied Physics, 32(5): 577-585. https://doi.org/10.1088/0022$3727 / 32 / 5 / 012$

[15] Miklavčič, M., Wang, C.Y. (2006). Viscous flow due to a shrinking sheet. Quarterly of Applied Mathematics, 64(2): 283-290. https://doi.org/10.1090/s0033-569x-0601002-5

[16] Wang., C.Y. (2008). Stagnation flow towards a shrinking sheet. International Journal of Non-Linear Mechanics, 43(5): 377-382. https://doi.org/10.1016/j.ijnonlinmec.2007.12.021

[17] Kumaran, V., Ramanaiah, G. (1996). A note on the flow over a stretching sheet. Acta Mechanica, 116(1-4): 229233. https://doi.org/10.1007/bf01171433

[18] Stewartson, K. (1951). On the impulsive motion of a flat plate in a viscous fluid, Part I. The Quarterly Journal of Mechanics and Applied Mathematics, 4(2): 182-198. https://doi.org/10.1093/qjmam/4.2.182

[19] Stewartson, K. (1973). On the impulsive motion of a flat plate in a viscous fluid. Part II. The Quarterly Journal of Mechanics and Applied Mathematics, 22(2): 143-152. https://doi.org/10.1093/qjmam/4.2.182

[20] Wang, C.Y., Du, Q., Miklavčič, M., Chang, C.C. (1997). Impulsive stretching of a surface in a viscous fluid. Siam Journal on Applied Mathematics, 57(1): 1-14. https://doi.org/10.2307/2951880
[21] Adomian, G. (1994). Solving Frontier Problems of Physics: The Decomposition Method. Springer Science + Business Media Dordrecht. B. V., Kluwer Academic Publishers, Dordrecht/ Boston/ London. https://doi.org/10.1007/978-94-015-8289-6

[22] Adomian, G. (1988). A review of the decomposition method in applied mathematics. Journal of Mathematical Analysis and Applications, 135(2): 501-544. https://doi.org/10.1016/0022-247X(88)90170-9

[23] Biazar, J., Shafiot, S.M. (2007). A simple algorithm for calculating Adomian polynomials. International Journal of Contemporary Mathematical Sciences, 2(20): 975-982.

[24] Cherruault, Y. (1990). Convergence of Adomian's method. Mathematical and Computer Modeling, 14: 8386. https://doi.org/10.1016/0895-7177(90)90152-D

[25] Cherruault, Y., Adomian, G. (1993). Decomposition methods: A new proof of convergence. Mathematical and Computer Modeling, 18(12): 103-106. https://doi.org/10.1016/0895-7177(93)90233-O

[26] DisuAkeem, B., Ajibola Saheed, O. (2014). Adomian Decomposition method for heat transfer and heat source in MHD flow over a stretching sheet. Mathematical Theory and Modeling, 4(12): 161-169.

[27] Baker, G.A., Graves-Morris, P. (1996). Pade approximants (2nd Edition). Encyclopedia of Mathematics and its Applications. Cambridge University Press, Cambridge.

[28] Boyd, J.P. (1997). Pade approximant algorithm for solving nonlinear ordinary differential equation boundary value problems on an unbounded domain. Computers in Physics, 11(3): 299-303. https://doi.org/10.1063/1.168606

[29] Williams, J.C., Rhyne, T.B. (1980). Boundary Layer development on a wedge impulsively set into motion. Siam Journal on Applied Mathematics, 38(2): 215-224. https://doi.org/10.2307/2101014

[30] Wellin, P. (2013). Programming with Mathematica: An Introduction (4th Revised Edition). Cambridge University Press, Cambridge.

[31] Liao, S.J. (2006). An analytic solution of boundary layer flows caused by an impulsively stretching plate. Communications in Nonlinear Science and Numerical Simulation, 11(3): 326-339. https://doi.org/10.1016/j.cnsns.2004.09.004

[32] Kechil, S.A., Hashim, I. (2007). Series solution for unsteady boundary layer flows due to impulsively stretching plate. Chinese Physics Letters, 24(1): 139-142. https://doi.org/10.1088/0256-307X/24/1/038

[33] Srinivasa, A.H., Eswara, A.T. (2011). Unsteady MHD laminar boundary layer flow due to an impulsively stretching surface. Proceedings of the World Congress on Engineering (WCE, 2011), London, UK, 1: 252-255. 